\title{
Velocidad de secado en tres tipos de secadores solares del Aguaymanto (Physalis Peruviana L.)
}

\author{
Drying rate of three types of solar dryers in \\ golden berry (Physalis Peruviana L.) \\ Camayo Lapa Bécquer Frauberth ${ }^{1 *} \quad$ Pablo Camargo Leonardo Ederson ${ }^{1}$ \\ Eguavil Tony Torres ${ }^{1}$ Juan Raúl Massipe Hernández ${ }^{2} \quad$ Marcos Quispe Flores $^{3}$ \\ Recibido 06 de marzo de 2018, aceptado 23 de diciembre de 2018 \\ Received: March 06, 2018 Accepted: December 23, 2018
}

\begin{abstract}
RESUMEN
En esta investigación, se planteó como objetivo determinar la velocidad de secado del Aguaymanto (Physalis Peruviana L.) en tres tipos secadores solares, para lo cual se diseñó y construyó los prototipos: directo, indirecto y mixto. El secado fue por radiación solar y convección natural de aire caliente generados por los colectores solares. Durante los ensayos se registraron las condiciones meteorológicas: la velocidad de viento media de $1,11 \mathrm{~m} / \mathrm{s}$, la humedad relativa del aire ambiente (HR) varió de $16 \%$ a $52 \%$ y la temperatura ambiental mínima y máxima fueron $14,7{ }^{\circ} \mathrm{C}(287,8 \mathrm{~K})$ y $26,4{ }^{\circ} \mathrm{C}(299,5 \mathrm{~K})$. La humedad inicial (bs) fue de 4,53 y la humedad final de 0,$07 ; 0,12$ y 0,10 ( $\mathrm{kg}$ agua $/ \mathrm{kg}$ sólido seco) respectivamente. Las velocidades de secado alcanzaron valores de: 0,0287; 0,0208 y 0,0407 ( $\mathrm{kg}$ agua /hora $\cdot \mathrm{m}^{2}$ ). El tiempo de secado: $41 \mathrm{~h}$ para el tipo directo, $56 \mathrm{~h}$ para el indirecto $56 \mathrm{~h}$ y $29 \mathrm{~h}$ del mixto. Los secadores solares es una alternativa viable, natural, económica y de fácil manejo para el deshidratado de los alimentos.
\end{abstract}

Palabras clave: Energía solar, secador solar, secado de aguaymanto, Physalis peruviana L.

\begin{abstract}
In this research, the objective was to determine the drying rate of aguaymanto (Physalis Peruviana $L$.) in three different solar dryers. To accomplish this aim three prototypes were designed and built direct, indirect, and mixed dryer. The drying process went through solar radiation and natural hot air convection generated by solar collectors. During rehearsals, weather conditions were recorded: average wind speed of $1.11 \mathrm{~m} / \mathrm{s}$, the relative air humidity $(\mathrm{RH})$ ranged from $16 \%$ to $52 \%$, and the minimum and maximum ambient temperature were $14.7{ }^{\circ} \mathrm{C}(287.8 \mathrm{~K})$ and $26.4{ }^{\circ} \mathrm{C}(299.5 \mathrm{~K})$. The initial moisture (bs) was 4.53 and the final moisture of 0.07; 0.12 and 0.10 ( $\mathrm{kg}$ water $/ \mathrm{kg}$ dry solid), respectively. Drying rates reached values: 0.0287; 0.0208 and $0.0407\left(\mathrm{~kg}\right.$ water $\left./ \mathrm{hour} \cdot \mathrm{m}^{2}\right)$. Drying time: $41 \mathrm{~h}$ for direct type, $56 \mathrm{~h}$ for indirect $56 \mathrm{~h}$, and $29 \mathrm{~h}$ mixed. Solar drying is a viable, natural, economical, and easy alternative to use for food de-hydration.
\end{abstract}

Keywords: Solar energy, solar dryers, drying aguaymanto, Physalis peruviana $L$.

\footnotetext{
1 Facultad de Ciencias Aplicadas. Universidad Nacional del centro del Perú. Aguirre Morales No 2096. Huancayo, Perú. E-mail: camayobecquer@hotmail.com; ecamargoleonardo@hotmail.com; tony_love80@hotmail.com

2 Alma Red Global. España. E-mail: raul.massipe@almaredglobal.com

3 naRural Energy. España. E-mail: moqf@ @otmail.com

* Autor de correspondencia: camayobecquer@hotmail.com
} 


\section{INTRODUCCIÓN}

El Perú es un lugar ideal para iniciar proyectos de energías renovables, debido a su gran abundancia de recursos y fuentes de energía verde: como el sol, el agua, la energía geotérmica, la eólica o la biomasa [1]. El uso de los sistemas térmicos solares para conservar vegetales y otras cosechas ha mostrado que es una opción práctica, económica y ambientalmente compatible. Los sistemas de calentamiento solar pueden mejorar la calidad del producto, a la vez que reduce desechos y combustibles tradicionales, mejorando la calidad de vida [2].

Los objetivos básicos que se pretenden con el uso de secadores solares, es mejorar el rendimiento del secado y minimizar el impacto ambiental [3]. Esta investigación propone estudiar la velocidad del secado del Aguaymanto en tres prototipos de secador, como se muestran en la Figura 1:

- el primero es un secador solar directo, que consiste en una cámara de secado donde el producto es expuesto directamente al sol;

- el segundo es un secador solar mixto que cuenta con cámara de secado expuesta al sol y además incluye un colector solar plano;

- el tercero es un secador solar indirecto con cámara de secado donde el producto no está expuesto directamente a los rayos solares además cuenta con colector solar plano propuestos por Espinoza [4].

Para el diseño y construcción se tuvo en cuenta los principios de transferencia de calor y masa, termodinámica y mecánica de fluidos. El Aguaymanto es una planta herbácea, perenne en zonas tropicales, que ejerce efectos antihiperglucémicos y, en consecuencia, pueden causar daño al tejido aliviador causado por la diabetes inducida por estreptozotocina [5]. Así mismo el extracto etanólico de sus hojas y tallos inhibe el crecimiento celular tumoral por la presencia de compuestos bioactivos [6].

Por lo que su cultivo es importante, ya que la cordillera de los andes peruanos ha contribuido a la gran diversidad de eco-tipos de Aguaymanto, base genética de los cultivos comerciales del mundo. Sin embargo, en el Perú, el cultivo en forma comercial es reciente. Se está cultivando en algunas zonas, como en Cajamarca, Cuzco, Huancayo, La Libertad y Ancash, especialmente para abastecer el mercado interno, y especialmente para el procesamiento con miras a la exportación (Programa de Desarrollo Rural Sostenible - cooperación alemana PDRS/ GIZ, 2011) citado por [7].

La contribución del trabajo consiste en estudiar la velocidad de secado del Aguaymanto (Physalis

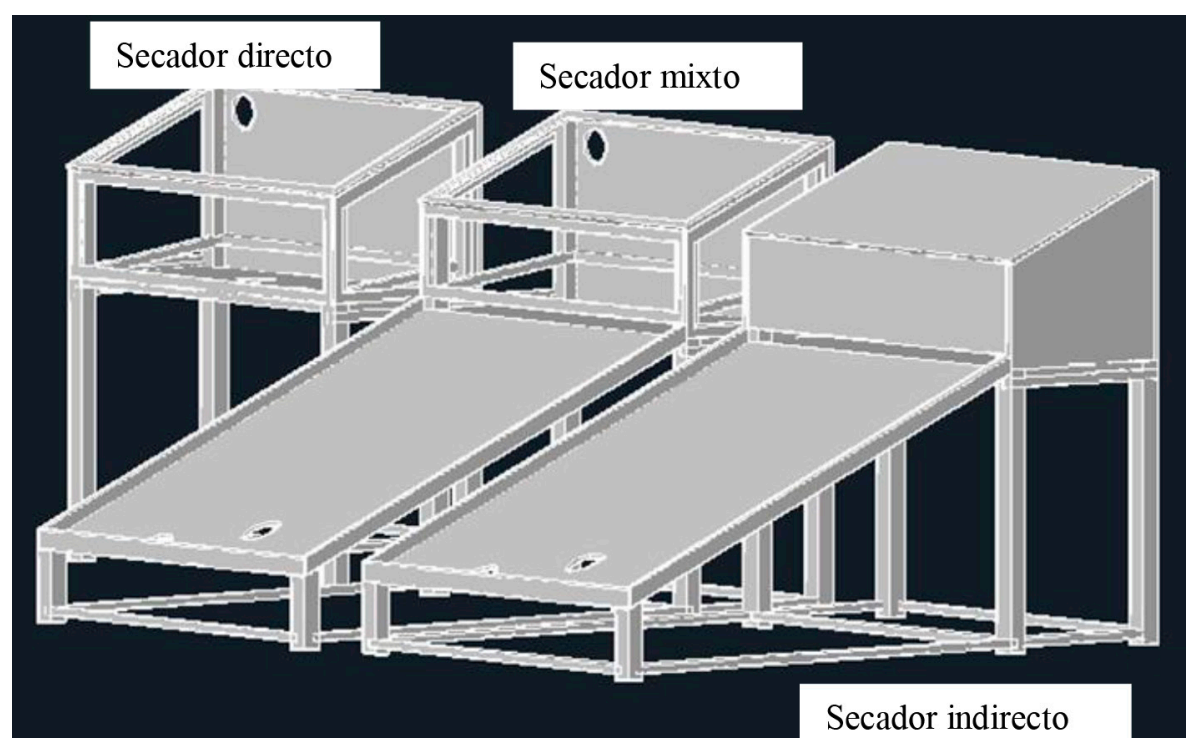

Figura 1. Prototipos de los 3 secadores diseñados. 
Peruviana L.) en los tres tipos secadores solares en las condiciones climáticas de Tarna y dar una alternativa viable, económica y natural a tratamientos convencionales, a los agricultores, especialmente, de la Región Junín, para el deshidratado del aguaymanto. Además, contribuir a disminuir la emisión de gases de efecto invernadero.

\section{MATERIALES Y MÉTODOS}

Para determinar la velocidad de secado, se diseñó y se construyó tres tipos de secadores solares, y así realizar el proceso de deshidratado desarrollando tecnología limpia, de una fruta funcional, como lo es el Aguaymanto (Physalis Peruviana.L.).

En el secado de alimentos es imprescindible conocer el tiempo necesario para deshidratar el producto hasta una determinada humedad final, con el objetivo de reducir el consumo energético, sin alterar la calidad del producto [8]; de allí la importancia de evaluar la velocidad de secado en los 3 prototipos construidos, lo cual nos ayuda a evaluar cuan eficientes son los secadores solares, y es por ello necesario conocer factores internos y externos que influyen en el proceso de secado. Entre los factores internos tenemos: humedad del producto, composición química y propiedades físicas del alimento, y entre los externos: humedad relativa del aire, temperatura, velocidad de aire, depresión del bulbo húmedo, área superficial y humedad de equilibrio.

El producto utilizado es el Aguaymanto, recolectado de uno, de las parcelas de Biocen Foods. Se trabajó con unos hidrómetros de $(0-100) \%$ humedad; estufa, Memmert UNE 200-800; balanzas de precisión de (0,001-1000) g; y termocuplas, de (0-180) ${ }^{\circ} \mathrm{C}$.

Para el diseño de los prototipos, se tomaron datos de la ciudad donde se está efectuó la investigación, es este caso Tarma, donde según [9], tiene las siguientes características climatológicas y geográficas: altitud de 3050 m.s.n.m., latitud de $11^{\circ} 25^{\prime} 12^{\prime \prime}$, y longitud de $75^{\circ} 41^{\prime} 17^{\prime \prime}$, con un clima árido y con sol casi todo el año, la velocidad media de viento es de $1,11 \mathrm{~m} / \mathrm{s}$, las precipitaciones anuales son de $600 \mathrm{a}$ $800 \mathrm{~mm}$, el promedio de temperatura es de $13{ }^{\circ} \mathrm{C}$ a $18{ }^{\circ} \mathrm{C}$ y la radiación solar anual promedio es de $5,43 \mathrm{k} * \mathrm{wh} / \mathrm{m}^{2} /$ día [10]. Para el cálculo de la cabina se consideró las características de la fruta como: el peso, diámetro y altura de $3 \mathrm{~cm}$.
Para el cálculo del incremento de la temperatura se utilizó la ecuación de cálculo termodinámico en donde una de las variables es la longitud del colector [4], y es:

Donde:

$$
\Delta T=(0,131 H) \cdot\left(1-e^{-0,12} / / v\right)
$$

$\Delta T$ : incremento de la temperatura del aire a través del colector, $\left[{ }^{\circ} \mathrm{C}\right]$;

$H$ : radiación solar, $\left[\mathrm{W} / \mathrm{m}^{2}\right] ; 1$; longitud en $\mathrm{m}$ y

$v$ : velocidad de aire en el colector, $[\mathrm{m} / \mathrm{s}]$.

Se determinó el ángulo de inclinación de colector solar en base a la ecuación considerado que nos encontramos en el hemisferio sur [11]:

$$
a=\delta-\varphi
$$

Donde:

$a$ : inclinación diaria del colector, [grados];

$\varphi$ : latitud, [grados];

$\delta$ : ángulo de declinación diaria del sol, [grados].

La determinación de la velocidad de secado y curvas de secado, el procedimiento se inició con análisis del contenido inicial de agua en base seca.

$$
H_{b s}=\frac{W_{0}}{W_{d}} \cdot 100
$$

Donde:

$H_{b s}$ : humedad en (bs), [kg agua/kg sólido seco];

$W_{0}$ : peso inicial de la materia sin secar, $[\mathrm{kg}]$;

$W_{d}$ : peso de la materia seca, $[\mathrm{kg}]$.

Por intermedio de una balanza, se pesó $1 \mathrm{~kg}$ de Aguaymanto, se colocó en los secadores solares, y se controló el tiempo de inicio de secado, se consideró la humedad libre del producto mediante la siguiente ecuación.

$$
X=X_{t}-X^{*}
$$

Donde:

$X_{t}$ : contenido de humedad, [kg agua/ $\mathrm{kg}$ sólido seco]; $X^{*}$ : humedad en equilibrio, [kg agua/ $\mathrm{kg}$ sólido seco].

El cálculo de la velocidad de secado, para cada hora, se calculó por la expresión [12]: 


$$
\begin{gathered}
t_{t s}=\frac{\lambda}{h_{c} A\left(T_{\alpha}-T_{s}\right)} \cdot\left(w_{a}-w_{c}\right)+ \\
\left.\left(w_{c}-w_{e}\right)\right] \cdot \operatorname{In} \frac{w_{c}-w_{e}}{w_{f}-w_{e}}
\end{gathered}
$$

Donde:

$t_{t s}:$ tiempo se secado total, [s];

$h_{c}$ : coeficiente convectivo de calor, $\left[\mathrm{W} / \mathrm{m}^{2 *} \mathrm{~K}\right]$;

$A$ : área, $\left[\mathrm{m}^{2}\right]$;

$T_{a}$ : temperatura bulbo seco, $\left[{ }^{\circ} \mathrm{C}\right]$;

$T_{s}$ : temperatura bulbo seco, $\left[{ }^{\circ} \mathrm{C}\right]$;

$\lambda$ : calor latente de vaporización, $[\mathrm{kJ} / \mathrm{kg}]$;

$w_{c}$ : humedad critica, [kg agua/kg sólido seco];

$w_{a}$ : humedad inicial, [kg agua $/ \mathrm{kg}$ sólido seco];

$w_{e}$ : humedad equilibrio, [kg agua/kg sólido seco];

$w_{f}$ : humedad final, [kg agua/kg sólido seco].

$$
R=\frac{L_{s}}{A} \frac{d X}{d t}
$$

Donde:

$R$ : velocidad de secado, $\left[\mathrm{kg} \mathrm{H}_{2} \mathrm{O} / \mathrm{hm}^{2}\right]$;

$L_{s}: \mathrm{kg}$ de sólido seco usado, $[\mathrm{kg}]$;

$A$ : es el área superficial expuesta al secado, $\left[\mathrm{m}^{2}\right]$.

Las mediciones por cada hora fueron: la pérdida de humedad del producto, Temperatura exterior y al interior de la cabina, humedad exterior e interior, se calculó la radiación médiate la ley de Stefan Boltzmann [13].

$$
Q=5,67 \cdot 10-8 \cdot \varepsilon \cdot T^{4}
$$

Donde:

$Q:$ Irradiación calorífica, $\left[\mathrm{W} / \mathrm{m}^{2}\right]$;

$\varepsilon$ : emisividad;

$T$ : temperatura, $[\mathrm{K}]$.

Mediante una hoja Excel se realizó las curvas de secado, asimismo se realizó el cálculo de velocidad promedio de toda la operación de secado hasta alcanzar un peso constante.

El diseño estadístico realizado fue el diseño completamente al azar (DCA), con tres tratamientos, el análisis de varianza ANOVA con $p>0,05 \%$ de significancia. Finalmente, se realizaron pruebas de comparación múltiple de Tukey al 95\% de para establecer las diferencias significativas. Los análisis se llevaron a cabo en la Universidad Nacional del Centro del Perú, en la Facultad de Ciencias Aplicadas de Tarma, en la Escuela Académica Profesional de Ingeniería Agroindustrial en los laboratorios de análisis de alimentos, de investigación frutas, hortalizas y de instrumentación.

\section{RESULTADOS Y DISCUSIÓN}

Las características en común de los prototipos son: volumen de la cabina de secado $1,20 \mathrm{~m}^{3}$, longitud del colector solar $1,30 \mathrm{~m}$, ángulo de inclinación $24^{\circ}$, dimensiones $1,80 \times 1,10 \times 1,50 \mathrm{~m}$, como se muestra en la Figura 2.

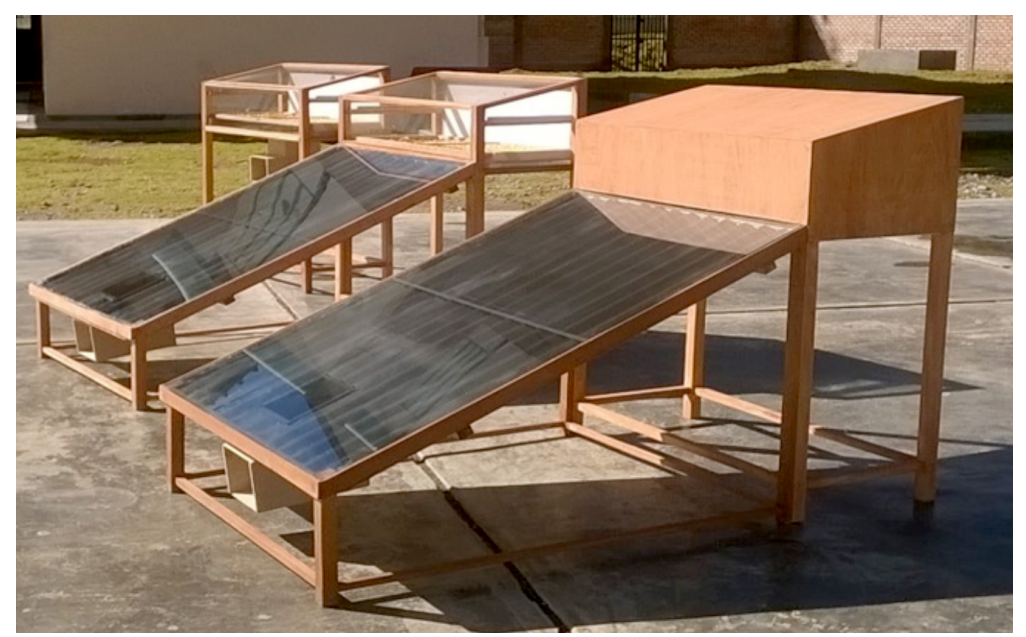

Figura 2. Prototipos de secadores solares construidos. 
De los tres tipos de secadores solares, el prototipo mixto fue el que mayor velocidad de secado alcanzo, con una velocidad de secado promedio mayor con respecto al secador indirecto de $0,0199 \mathrm{kgH}_{2} \mathrm{O} / \mathrm{hm}^{2}$. Así mismo la temperatura alcanzada por este secador, se diferencia significativa de los otros dos. En la Tabla 1, se puede observar los diferentes resultados obtenidos y sus diferencias. La humedad inicial (bs) fue de 4,53 y la humedad final de 0,07; 0,12 y 0,10 (kg agua $/ \mathrm{kg}$ sólido seco) respectivamente. Las velocidades de secado en los secadores solares indirecto, directo y mixto llego a: 0,0287; 0,0208 y $0,0407\left(\mathrm{~kg}\right.$ agua $\left./ \mathrm{hora} \cdot \mathrm{m}^{2}\right)$ respectivamente.

La humedad decreciente en función del tiempo de los prototipos presento oscilaciones por la variación de la temperatura en cada hora, este fenómeno puede ser controlado por diseños de tipo híbridos, que se pudiera controlar la velocidad de ingreso y salida de aire automáticamente, la automatización debe ser analizado por las siguientes investigaciones.
Durante los ensayos se registraron las condiciones meteorológicas: la velocidad de viento media de $1,11 \mathrm{~m} / \mathrm{s}$, la humedad relativa del aire ambiente (HR) varió de $16 \%$ a $52 \%$ y la temperatura ambiental mínima y máxima fue de $14,7{ }^{\circ} \mathrm{C}$ $(287,8 \mathrm{~K})$ y $26,4{ }^{\circ} \mathrm{C}(299,5 \mathrm{~K})$. Como se muestra en la Figura 3, el tiempo de secado alcanzo: $41 \mathrm{~h}$ para el tipo directo, $56 \mathrm{~h}$ para el indirecto $56 \mathrm{~h} \mathrm{y}$ $29 \mathrm{~h}$ del mixto.

En la Figura 4, se muestra las pruebas de comparación múltiple para el tiempo de secado que mostraron que hubo una diferencia significativa del secador tipo mixto y directo con respecto al prototipo indirecto, ya que presentó una media de secado mayor de los otros dos secadores.

Otra diferencia significativa la tuvimos en la temperatura de la cámara de secado como se muestra en la Figura 5. Se observó que la temperatura de cámara en el secador solar mixto es significativamente

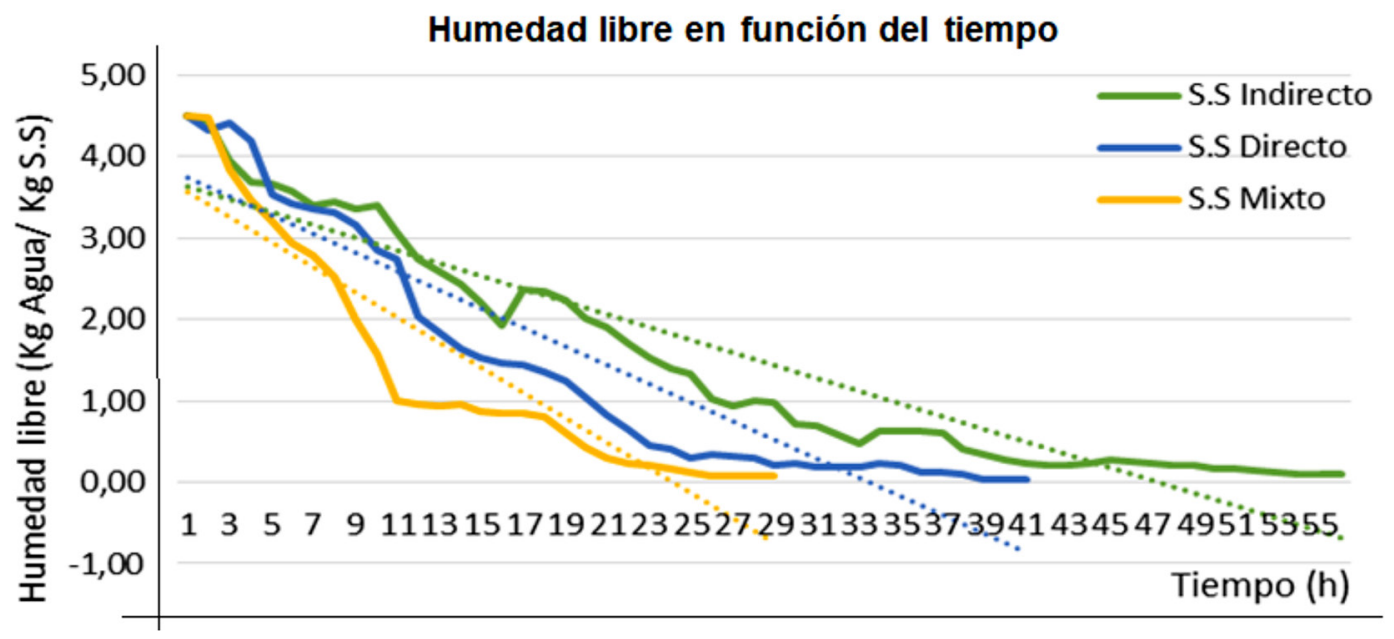

Figura 3. Pérdida de la humedad libre en función del tiempo en los secadores solares.

Tabla 1. Resultados de la investigación.

\begin{tabular}{|l|c|c|c|c|}
\hline \multicolumn{1}{|c|}{ Prototipo } & Unidad Medida & Indirecto & Directo & Mixto \\
\hline Humedad & $(\mathrm{kg} \mathrm{Agua} / \mathrm{kg} \mathrm{S.S.})$ & $4,51-0,09$ & $4,51-0,04$ & $4,51-0,07$ \\
\hline Tiempo de Secado & $(\mathrm{Horas})$ & 56 & 41 & 29 \\
\hline Velocidad Secado & $\left(\mathrm{kg} \mathrm{H}: \mathrm{O} / \mathrm{h} \cdot \mathrm{m}^{2}\right)$ & 0,0208 & 0,0287 & 0,0407 \\
\hline Peso Constante & $(\mathrm{kg})$ & 0,202 & 0,192 & 0,198 \\
\hline Temperatura Ambiental & $\left({ }^{\circ} \mathrm{C}\right)$ & $14,7-26,3$ & $14,7-25,7$ & $14,7-25,7$ \\
\hline Humedad Relativa & $(\%)$ & $16-52$ & $16-52$ & $16-52$ \\
\hline Temperatura Cabina & $\left({ }^{\circ} \mathrm{C}\right)$ & 65,2 & 65,3 & 88,8 \\
\hline
\end{tabular}


COMPARACIÓN MÚLTIPLE TUKEY DEL TIEMPO DE SECADO 28,500 (b)

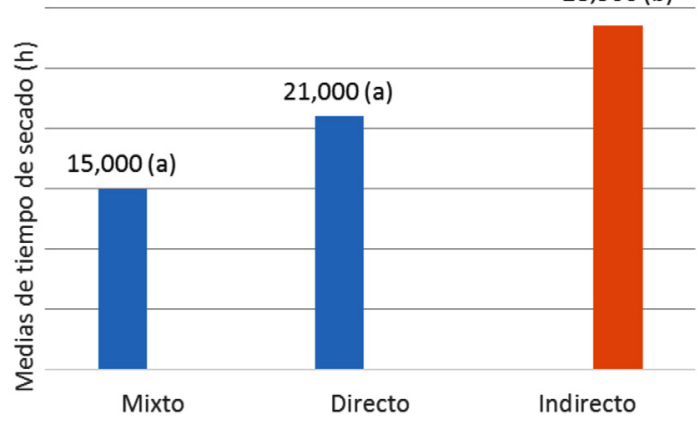

Figura 4. Prueba de comparación múltiple Tukey para el tiempo de secado.

\section{COMPARACIÓN MÚLTIPLE TUKEY DE LA TEMPERATURA DE CABINA.}

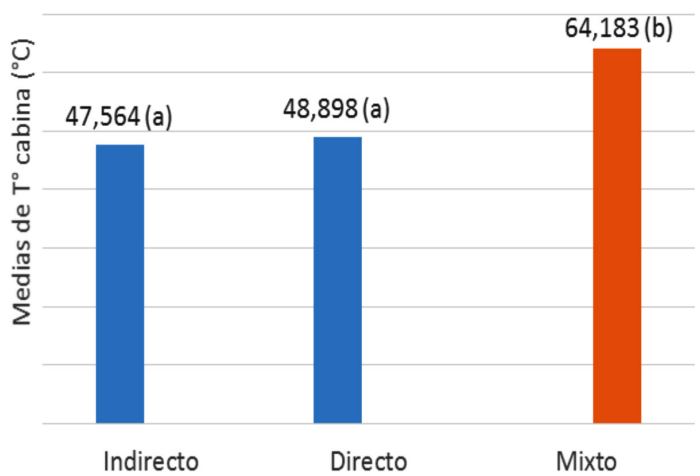

Figura 5. Prueba de comparación múltiple Tukey para la temperatura de la cámara.

mayor con respecto a los otros dos prototipos debido al mayor aporte combinado de energía: la directa a la cámara y la del colector solar plano.

Así mismo no se mostraron diferencias significativas entre la velocidad de secado y el descenso de la pérdida de humedad entre los prototipos.

En el secado de cascara de piña con una humedad de $80 \%$ de humedad con un modelo indirecto presento una velocidad promedio de $0,131 \mathrm{~kg} . \mathrm{H}_{2} \mathrm{O} / \mathrm{hm}^{2}$ [12], así mismo en la deshidratación de plátanos por secadores tipo mixto mostrados por [15], muestra una velocidad de secado de $0,049 \mathrm{kgH}_{2} \mathrm{O} / \mathrm{hm}^{2}$, que en comparación con nuestros resultados tiene el mismo orden de magnitud.
Uno de los resultados a resaltar es que tanto el secador solar mixto como el secador solar directo han alcanzado temperaturas mayores que $70^{\circ} \mathrm{C}$ en la cámara de secado lo cual es recomendable para frutos que solo se busca por su contenido de fibra más no por mantener la vitamina C. En cambio el secador solar indirecto, cuya velocidad de secado es menor, en su cámara de secado llega a temperaturas menores de $65^{\circ} \mathrm{C}$, lo cual hace que se cumpla los requisitos para mantener las cualidades nutricionales de las frutas, lo que nos permite deshidratarlas para el mercado nacional e internacional, lo que corrobora Morales [15] que concluye que es posible desarrollar tecnología apropiada para el secado de productos agrícolas en el municipio de Acapetuha, Chiapas, en su caso se realizó el diseño, cálculo y construcción de un secador solar indirecto.

\section{CONCLUSIONES}

Los resultados expuestos y descritos, nos permite llegar a las conclusiones siguientes:

- Se ha estudiado y determinado la velocidad de secado del Aguaymanto (Physalis Peruviana L.) en tres tipos secadores solares en las condiciones climáticas de Tarma que no se diferencian significativamente de los tratamientos convencionales de deshidratado que es secado con energía eléctrica y/o gas.

- Se ha obtenido valores medios de la velocidad de secado del Aguaymanto para los secadores solares indirecto, directo y mixto de 0,0208 $\mathrm{kgH}_{2} \mathrm{O} / \mathrm{hm}^{2}, 0,0287 \mathrm{kgH}_{2} \mathrm{O} / \mathrm{hm}^{2}$ y 0,0407 $\mathrm{kgH}_{2} \mathrm{O} / \mathrm{hm}^{2}$, respectivamente;

- Se ha demostrado que la tecnología de secado solar es una alternativa viable, económica y natural a los deshidratadores convencionales para los agricultores, especialmente, de la Región Junín, para el deshidratado del Aguaymanto;

- Existen diferencias significativas entre el tiempo y temperatura de secado de las cámaras de los prototipos estudiados, se ha obtenido los mejores resultados en el secador solar mixto;

- Para una radiación solar máxima de 619 W $\mathrm{h} / \mathrm{m}^{2}$, las temperaturas máximas alcanzas dentro de la cámara de secado para los secadores indirecto, directo y mixto fue de: $65,2{ }^{\circ} \mathrm{C}$; $65,3^{\circ} \mathrm{C}$ y $88,8^{\circ} \mathrm{C}$, respectivamente. Por lo que recomendamos secar a temperaturas menores de $65^{\circ} \mathrm{C}$ para mantener mayores cantidades de 
Vitamina $\mathrm{C}$ y para consumir como fibra puedes deshidratarse a mayores temperaturas.

\section{REFERENCIAS}

[1] P. Gamio Ayta. "Energías Renovables y cambio climático". Konrad Adenauer Stiftung. Lima, Perú. 2015.

[2] L. Roche-Delgado, J.P. Hernández-Touset y A. García-Rodríguez. "Diseño conceptual de secador solar a escala piloto para algas marinas". $R T Q$ [online]. Vol. $37 \mathrm{~N}^{\circ} 2$ [citado 2017-10-10], pp. 184-200. ISSN: 2224-6185. 2017.

[3] J.A. Palacio-F., B.E. Cadavid y D.E. Agudelo. "Sistema tipo marquesina de doble cámara, para el deshidratado de plantas aromáticas y medicinales empleando energía solar". Revista Politécnica. Vol. $12 \mathrm{~N}^{\mathrm{o}}$ 22, pp. 51-55. 2016.

[4] R. Espinoza. "Tecnología del secado solar". $1^{\text {ra }}$ Edición, Editorial ingeniería SA., Lima, Perú. 2001.

[5] F. Erman, T. Kaya, O. Yilmaz, O. Erman and A.D. Ozsahin. "Influences of Physalis Peruviana L. and Lupinus Albus L. Extracts on the levels of some biochemical parameters in Erythrocytes and Serum of Streptozotocin induced diabetic rats". Fresenius Environmental Bulletin. Vol. 26, Issue 7, pp. 4876-4882. July, 2017.

[6] D. Zavala, A. Quispe, M. Posso, R. Rojas and A. Vaisberg. "Efecto citotóxico de Physalis peruviana (capulí) en cáncer de colon y leucemia mieloide crónica". Readylic org. ISSN: 1025-5583. 2006.

[7] G. Fischer, P.J. Almanza-Merchan e D. Miranda. "Importância e cultivo do (Physalis peruviana L.) no mundo. Rev. Bras. Frutic. [online]. Vol. $36 \mathrm{~N}^{\mathrm{o}}$ 1, pp. 01-15. 2014.
[8] R. Giraldo, T. Vargas y H. Gil. "Mejoramiento del proceso de deshidratación de Uchuva". Revista Publicaciones e Investigación. Vol. 3 No 1. ISNN: 1900-6608. Agosto 2009.

[9] Instituto Geográfico Nacional. "El Geógrafo". Revista técnico-científica del Instituto Geográfico Nacional. Publicado el 20 de febrero de 2016. 2011.

[10] B.F. Camayo Lapa et al. "Validación y aplicación del modelo Bristow Campbell para estimar la radiación solar global de la región de Junin". $R T Q$ [online]. Vol. $37 \mathrm{~N}^{\circ} 3$ [citado 2017-1009], pp. 574-590. ISSN: 2224-6185. 2017.

[11] A. Veldecir, D. Marcal, J. Marques y R. Sinicio. "Secado de granos natural, solar y a bajas temperaturas". $1^{\text {ra }}$ Edición, Santiago de Chile. 1991.

[12] C. Geankoplis. "Proceso de transporte y operaciones unitarias". México, continental. p. 579.1998.

[13] V. Romero Rochín. "Termodinámica del Cuerpo Negro y la Ley de Stefan-Boltzmann". 2010. Artículo encontrado en: http://www. fisica.unam.mx/personales/romero/2010/ Cuerpo-Negro.pdf

[14] D. Muños y G. Cabrera. "El secado directo e indirecto de Piña". Revista Biotecnological. Vol. $4 \mathrm{~N}^{\circ}$ 1. 2006. URL: http://revistabiotecnologia.unicauca.edu. co/revista/index.php/biotecnologia/article/ view/31/20

[15] F. Ramirez y A. Vergara. "Proceso de deshidratado solar en plátanos". RSA - 1201. 2008.

[16] M.A. Morales Ovando, E. Mejía de los Santos, Á.S. Ángeles Escobar, M.E. Domínguez Espinosa y J. López Robler. "Cálculo y diseño de dos secadores solares con sistema fotovoltaico para deshidratación de alimentos". Lacandonia, Año 10. Vol. 10, $\mathrm{N}^{\mathrm{o}}$ 1, pp. 87-90. Junio de 2016. 